\title{
Deductive Prediction of Measurement Precision from Signal and Noise in Fluorometry
}

\author{
Yuzuru HaYashi ${ }^{\dagger}$ and Rieko Matsuda \\ National Institute of Health Sciences, Kami-Yoga, Setagaya, Tokyo 158, Japan
}

\begin{abstract}
The aim of this paper is to examine the precision of fluorometry on the basis of a recently proposed theory to predict the relative standard deviation (RSD) of measurements from signal and noise in an analytical instrument. According to the theory, the instrumental baseline drift which is often formulated as $1 / f$ noise can well be approximated by a mixed random process of white noise and the Markov process. The standard deviations (SD), $\widetilde{w}$, of the white noise and the SD, $\widetilde{m}$, and auto-correlation parameter, $\rho$, of the Markov process can be parametrized from the power spectral density of the blank line drift by the least squares curve fitting. All the parameters necessary for the uncertainty prediction $(\widetilde{w}, \widetilde{m}, \rho$, signal domain, $\left(k_{\mathrm{c}}+1, k_{\mathrm{f}}\right)$ and signal area, $A$, over the domain), can be determined directly and uniquely from experiments and experimental design. No arbitrary constants are involved in the prediction theory. The uncertainty prediction is shown to be superb over a wide concentration range of Rhodamin B in the fluorescence measurement. The influence of the integration domain on the precision is considered.
\end{abstract}

Keywords Fluorometry, precision, $1 / f$ noise; Markov process

The precision of measurements which is usually expressed in statistical terms as RSD is of great importance in theory and practice in analytical chemistry. ${ }^{1}$ If the precision can be predicted from the operating conditions of an analytical instrument without repeating experiments, its application will be fruitful in quantitative analysis. For example, the analytical performance of a newly developed method as well as diverse traditional methods can be evaluated with reference to the universal criterion (precision). In limited situations of HPLC, the mobile phase composition can be optimized to select the optimum which provides the highest precision from among all the conditions examined. ${ }^{2}$

Due to its invaluable significance, review articles and tutorials have been published concerning the theoretical aspects of the precision in HPLC ${ }^{2,3}$, and spectrometry. ${ }^{4-13}$ As for the precision in fluorometry, however, not many papers could be found during these two decades. ${ }^{14-16}$ Quite recently, a new theory to predict the actual RSD values in analytical instruments has been proposed and applied to an HPLC apparatus equipped with a photomultiplier. ${ }^{17}$ This uncertainty prediction was excellent for peaks of various widths, heights and areas over a wide concentration range of some aromatic compounds.

The prediction theory can be characterized by the following properties: $:^{17}$

(1) the baseline drift is approximated by a mixed random process of white noise and the Markov process and the

† To whom correspondence should be addressed. actual RSD values of measurements in analytical instruments can be calculated from the signal shape and stochastic properties of the random process;

(2) all the parameters necessary for the uncertainty prediction can be determined directly and uniquely from experiments and no arbitrary constants are involved. The baseline drift is often formulated as $1 / f$ noise $(f$ is frequency). ${ }^{18} \quad A$ surprisingly large number of phenomena in nature have been identified as a $1 / f$ fluctuation. ${ }^{19}$

Some readers would expect the well-known dependence of the precision on the signal-to-noise ratio of instrumental baseline drifts, i.e., a $1 / \sqrt{n}$ dependence where $n$ denotes the number of measurements or samples. The practical IUPAC recommendation of the definition of the limit of detection (LOD) in HPLC concerns the $S / N$ ratio $^{20}$, although the general IUPAC definition is $33 \%$ RSD of measurements. ${ }^{21}$ If the baselines are timeindependent like the white noise, the $S / N$ ratio could mathematically be related to the precision by means of the range in statistical terminology. ${ }^{22}$ However, actual HPLC baselines are strongly time-correlated ${ }^{17,18}$ and the precision in the apparatus cannot be predicted straightforwardly from the $1 / \sqrt{n}$ dependence. The auto-correlation of the baselines is the most essential reason why the above theory is required for the exact prediction of the instrumental precision and determination of LOD. ${ }^{23}$ The theory is clearly different in principle from the others mentioned above. ${ }^{4-13}$

The aim of this paper is to apply the prediction theory to the fluorescence determination of Rhodamin B. Although the effectiveness of the theory has only been 
proved for a photomultiplier and photodiode detector in HPLC ${ }^{17,24}$, the substantial applicability of the prediction theory is discusssed.

\section{Materials and Experiments}

Rhodamin B was purchased from Kishida Chemicals \& Co. Ltd. The original solution was $100 \mu \mathrm{g} / 1$ of Rhodamin B in Milli Q water. Thousand-, 500-, 200and 50-fold diluted solutions of the original solution were used for the measurement.

The fluorescence was measured with a Hitachi 650-60 fluorescence spectrometer equipped with Ushio xenon short arc lamp (Type UXL-157). The excitation and emission wavelengths were 556 and $640 \mathrm{~nm}$, respectively, with a bandpass of $5 \mathrm{~nm}$. A one-centimeter quartz fluorescence cell was used for the single-channel intensity measurement. The voltage of the photomultiplier tube was set at the middle range of the instrument. A computerized data handling system was used: the sampling intervals of the analog-to-digital converter was $200 \mathrm{~ms}$ and the low-pass filter of an amplifier was tuned at $10 \mathrm{~Hz}$. The apparatus was placed in an airconditioned room, and no temperature controller was installed for the cell. All the measurements were made after a long warming-up period (about $12 \mathrm{~h}$ ). The cell orientation (face of the cell closest to the excitation radiation) was held constant.

Figure 1 illustrates the time course of the fluorescence measurement for Rhodamin B. The fluorescence signal appears just after the shutter for the lamp is opened and disappears after it is closed. The datum marked with Zero is adopted as the zero signal intensity and the integration of the raw data is started $3 \mathrm{~s}$ (15 data points) after the zero point. The shutter is opened for $20 \mathrm{~s}$. This measurement called measurement 1 results in the data integrated up to $0.2,0.4, \cdots, 16 \mathrm{~s}$ (the sampling intervals of the A/D converter is fixed at $0.2 \mathrm{~s}): S(1,0.2)$, $S(1,0.4), \cdots, S(1,16)$. The precision of measurements is obtained by repeating the above measurement $n$ times. For example, the RSD for an integration time of $1.4 \mathrm{~s}$ is calculated in the usual statistical way from the $n$ experimental data, $S(1,1.4), S(2,1.4), \cdots, S(n, 1.4)$. In this study, ten replicate measurements were made of each solution $(n=10)$.

The blank data were obtained in the same way as the samples, but a 20-point integration was used for correcting the fluorescence signals. After subtracting the average blank signal, the above RSDs were calculated. The power spectral density of the blank line was obtained from the data collected by continuously monitoring the light from the reference solution over a period of $819.2 \mathrm{~s}$ (4096 data points).

As is indicated by Almeida and Seitz ${ }^{16}$, the long-time exposure of a sample against the excitation light decreases the fluorescence intensity because of the formation of a thermal lens caused by the nonradiative decay of excited states of a fluorophor. This intensity

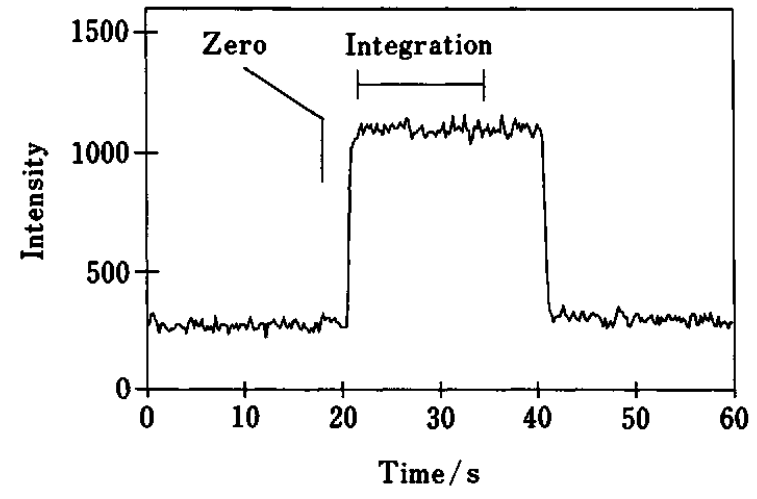

Fig. 1 Time course of fluorescence measurement. The concentration of the sample is $1 \mu \mathrm{g} / \mathrm{l}$.

change leads in turn to serious error in the integration measurements. While the shutter is closed between the measurements, the sample solution was exchanged with the new one of the same concentration at room temperature with the cell kept in the holder. For the sample of the highest concentration $(2 \mu \mathrm{g} / 1)$, the cell was removed from the holder for the sample exchange. None of the samples were stirred.

The effect of the waiting time ( $=k_{\mathrm{c}}$ data points) and integration time on the precision is also examined by the Monte Carlo simulation. If the integration is conducted from $k_{\mathrm{c}}+1$ to $k_{\mathrm{f}}$, then the number of data points in the integration domain is $k_{\mathrm{f}}-k_{\mathrm{c}}$. The waiting time is fixed at $3 \mathrm{~s}$ in the fluorescence measurements mentioned above.

\section{Theory}

A brief review of the prediction theory is given here for a better understanding of this paper. The detailed explanation can be found in the first paper of this study. ${ }^{17}$ The precision of measurements can be predicted from signal and noise parameters. The signal parameters include the waiting time, $k_{\mathrm{c}}$, integration time (or data points), $k_{\mathrm{f}}-k_{\mathrm{c}}$, and signal area (or the average of the summed signal intensities), $A$, over the integration time $\left(k_{r}-k_{\mathrm{c}}\right)$. If the signal height is $h$, the signal area is $h \times\left(k_{\mathrm{f}}-k_{\mathrm{c}}\right)$. After the baseline drift is Fouriertransformed into the power spectral density, the noise parameters ( $\widetilde{w}, \widetilde{m}$ and $\rho$ ) can be determined by the least squares curve fitting of the power density (see below). The noise parameters are indigenous to an instrument itself and are common to all the samples.

The stochastic structure of the white noise and Markov process has been studied in much more detail in mathematics $^{25}$ than the $1 / f$ noise. The following equation is used for predicting the fluorescence precision (for the derivation, see Appendix):

$$
\operatorname{RSD}^{2}=\frac{\left(k_{\mathrm{f}}-k_{\mathrm{c}}\right) \widetilde{w}^{2}}{A^{2}}+\frac{1}{(\tilde{\imath}-\rho)^{2}} \times
$$




$$
\begin{aligned}
& \left(k_{\mathrm{f}}-k_{\mathrm{c}}-2 \rho \frac{1-\rho^{k \mathrm{f}-k \mathrm{c}}}{1-\rho}+\rho^{2} \frac{1-\rho^{2(k \mathrm{f}-k \mathrm{c})}}{1-\rho^{2}}\right) \frac{\widetilde{m}^{2}}{A^{2}} \\
& +\rho^{2} \frac{1-\rho^{2 k \mathrm{c}}}{1-\rho^{2}}\left(\frac{1-\rho^{k \mathrm{f}-k \mathrm{c}}}{1-\rho^{2}}\right) \frac{\widetilde{m}^{2}}{A^{2}}
\end{aligned}
$$

The first term of Eq. (1) denotes the statistical contribution of the signal and white noise and the second and third terms that of the signal and Markov process. The third term becomes zero, if the waiting time is zero $\left(k_{\mathrm{c}}=0\right)$. Therefore, it describes the influences of the waiting time on the precision. The first and second terms denote the waiting-free effect of the mixed random process exclusively over the integration domain $\left(k_{\mathrm{c}}+1\right.$, $k_{\mathrm{f}}$ ), because every time variable refers to the difference $\left(k_{\mathrm{f}}-k_{\mathrm{c}}\right)$.

The power spectrum of the mixed process of the white noise and Markov process can be described as the sum of the power spectra of the individual processes: ${ }^{17}$

$$
P(k)=\frac{2 \widetilde{m}^{2}}{(1+\rho)\left[(1-\rho)^{2}+4 \pi^{2}(k / N)^{2}\right]}+\widetilde{w}^{2}
$$

where $N$ denotes the total number of data points collected for the blank line drift, but $k$ denotes a "frequency" in the power spectral density $(k=0,1, \cdots, N / 2)$. The actual frequency (cps) is $k /(\Delta T N)$ where $\Delta T$ denotes the sampling intervals of the A/D converter used. The first term of Eq. (2) concerns the Markov process characterized by $\widetilde{m}$ and $\rho$ and the second term that of the white noise with an SD of $\tilde{w}$. The power density of the white noise is constant, but that of the Markov process is Lorentzian. ${ }^{25}$ The noise parameters, $\widetilde{w}, \widetilde{m}$ and $\rho$, can be determined by doing the least squares fitting toward the actual power spectral density of the blank line drift with $P(k)$ used as a model curve.

In a Markov process, a random noise, $r(t+\Delta t)$, at time $t+\Delta t$ retains a part of the previous state, $r(t)$, at time $t$ with a retention ratio of $\rho(-1<\rho<1)::^{25}$

$$
r(t+\Delta t)=\rho r(t)+m(t)
$$

In this paper, $m(t)$ is assumed to be white noise at time, $t$, with the SD of $\widetilde{m}$. This white noise, $m(t)$, is probabilistically independent of the above-mentioned white noise. If $\rho=0$, then the Markov process is equivalent to the white noise with an SD of $\widetilde{m}$.

\section{Monte Carlo Simulation of Fluorometry}

Before drawing up a plan of experiments in this paper, it is beneficial to probe the statistical aspects of fluorometry with Monte Carlo simulation. Figure 2 shows the effect of the integration domain on the precision of fluorescence measurements with the waiting time fixed at zero. The bottom line and symbol (O) denote the theoretical RSD and the results of the Monte Carlo simulation, respectively, in the situation where the

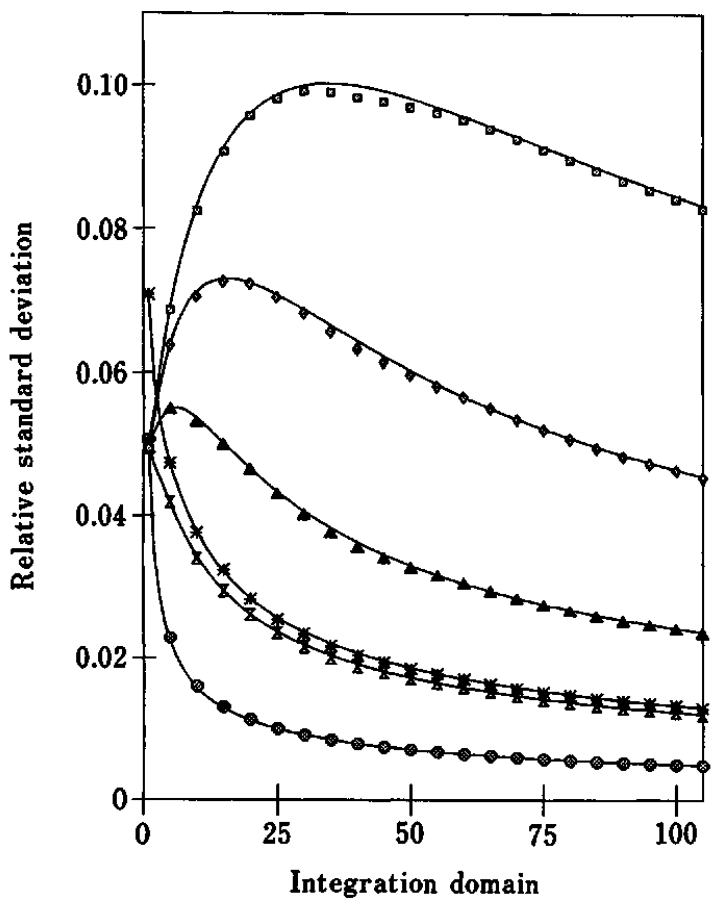

Fig. 2 Dependence of precision of measurements on noise and integration time (Monte Carlo simulation). The abscissa denotes the data points over which the raw data are integrated. The solid lines were obtained by Eq. (1). The symbols denote the results from the Monte Carlo simulation. $\square: \widetilde{w}=0, \widetilde{m}=5, \rho=0.95 ; \diamond: \widetilde{w}=0, \widetilde{m}=5, \rho=0.9 ; \Delta: \widetilde{w}=0$, $\widetilde{m}=5, \rho=0.8 ; \nabla: \widetilde{w}=0, \widetilde{m}=5, \rho=0.6 ; \bigcirc: \widetilde{w}=5, \widetilde{m}=0 ; *: \widetilde{w}=5$, $\widetilde{m}=5, \rho=0.6$. Signal height is fixed at 100 . $n=5000$.

white noise is the only error source in the drifts of blank signal $(\widetilde{w}=5 ; \widetilde{m}=0)$. As the integration domain increases, the RSD of measurements decreases monotonically. This improvement in the precision is attributable to a well-known reason, i.e., the accumulation of data by repeated measurements or the $S / N$ ratio.

The statistical influence of the Markov process is known from the upper lines and symbols. If the integration domain is fixed at a value, the RSD of measurements increases with increasing auto-correlation parameter, $\rho$, of the Markov process $(\mathbb{Z}, \Delta, \diamond, \square)$. If the auto-correlation parameter is small (see $\times$ for $\rho=0.6$ ), the precision has a similar trend toward the integration to that for the white noise (O). However, if $\rho \geq 0.8(\square, \diamond$, $\triangle$ ), the RSD increases first and then decreases with increasing integration domain.

The increase in the RSD with increasing autocorrelation parameter is due to the waves of long cycles involved in the blank line. Suppose that a wave has a cycle much longer than the signal width (here, the integration domain). A rectangular signal lying on such a wave will be seriously deformed and the observed signal shape will greatly vary depending on the location of the signal on the wave, i.e., a ridge or valley. A large autocorrelation parameter means a high possibility of the low frequency waves existing in the blank line or strong timecorrelation of the baseline. Low frequencies existing in 


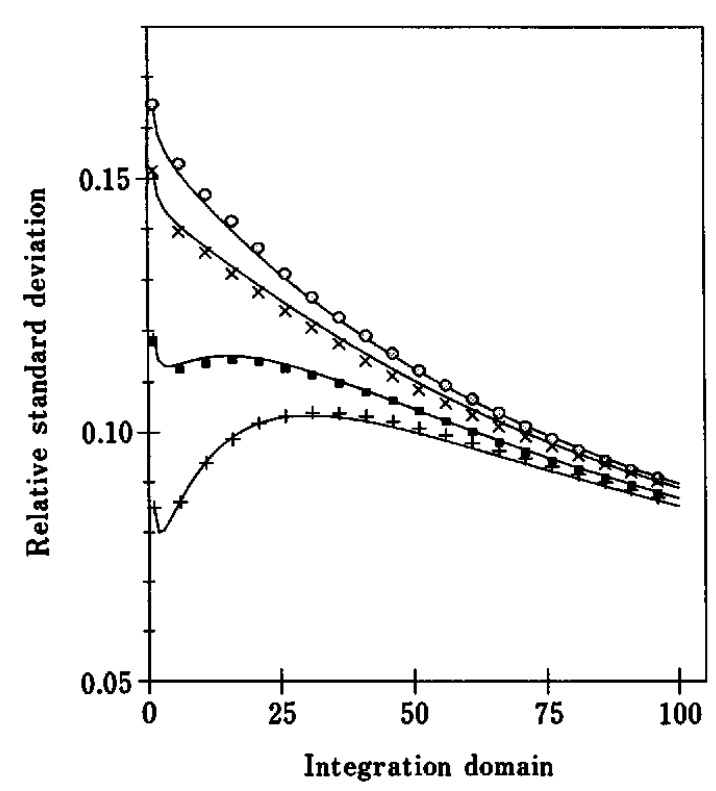

Fig. 3 Influence of waiting time on precision of measurement (Monte Carlo simulation). The abscissa denotes the data points over which the raw data are integrated. The solid lines are obtained by Eq. (1). Symbols denote the results from the Monte Carlo simulation. $\widetilde{w}=0, \widetilde{m}=5$ and $\rho=0.95$. $0: k_{\mathrm{c}}=1 ; \times: k_{\mathrm{c}}=5 ; \square: k_{\mathrm{c}}=15 ;+: k_{\mathrm{c}}=30$. The signal height is fixed at 100 . $n=5000$.

HPLC baselines have already been observed ${ }^{17}$ (also see Fig. 4). In this situation, therefore, we can easily imagine that the larger is the auto-correlation parameter, the more is the scattering of the observed area. In Fig. 2, the RSD of measurements for 50 integration points is $c a$. 0.1 for $\rho=0.95$ and 0.3 for $\rho=0.8$. On the other hand, a great number of positive and negative parts of the waves of short cycles can be canceled out over the relatively long integration domain, although the signal contaminated by the low frequencies looks ragged.

The line and symbol (*) shows the RSD obtained from the mixed random process of the white noise $(O)$ plus the Markov process $(X)$. The statistical effect of the white noise is not so important as that of the Markov process. The waves of long cycles involved in the Markov process enhance the imprecision more than do the waves of the short cycles as mentioned above. This is the reason why the effect of the long waves (or the time-correlation described by the Markov process) should be included in the theory for the exact prediction of the instrumental uncertainty.

In Fig. 2, the integration begins just after the zero point, i.e., the waiting time is zero $\left(k_{\mathrm{c}}=1\right)$. Figure 3 demonstrates the effect of the waiting time on the precision in case of a large auto-correlation parameter $(\rho=0.95)$. If the integration time is fixed, the RSD of fluorescence measurements increases with increasing waiting time. As long as the waiting time is small (+), the RSD behavior against the integration resembles that with zero waiting time shown in Fig. 2.

The zero waiting time is unrealistic because of the finite time constant of the detection unit. The output of the unit used here took about two seconds to reach the stable state after the shutter was opened. The waiting time for measurement more than $k_{\mathrm{c}}=30$ data points exerts an additional small influence on the precision. For example, the theoretical RSD for the peak-height measurement $\left(k_{\mathrm{f}}-k_{\mathrm{c}}=1\right)$ is $16.45 \%$ for a realistic waiting time $\left(k_{\mathrm{c}}=30\right)$ and $16.78 \%$ for a much longer waiting time $\left(k_{\mathrm{c}}=100\right)$.

\section{Precision of Fluorometry}

Figure 4A illustrates an example of the blank line drift observed in the fluorometer used and the zigzag line of Fig. 4B is its power spectral density. The smooth line of Fig. 4B is drawn according to Eq. (2). The noise parameters were determined from a blank line drift of 4096 data points $(\widetilde{w}=17.3, \widetilde{m}=5.57$ and $\rho=0.955)$, but for
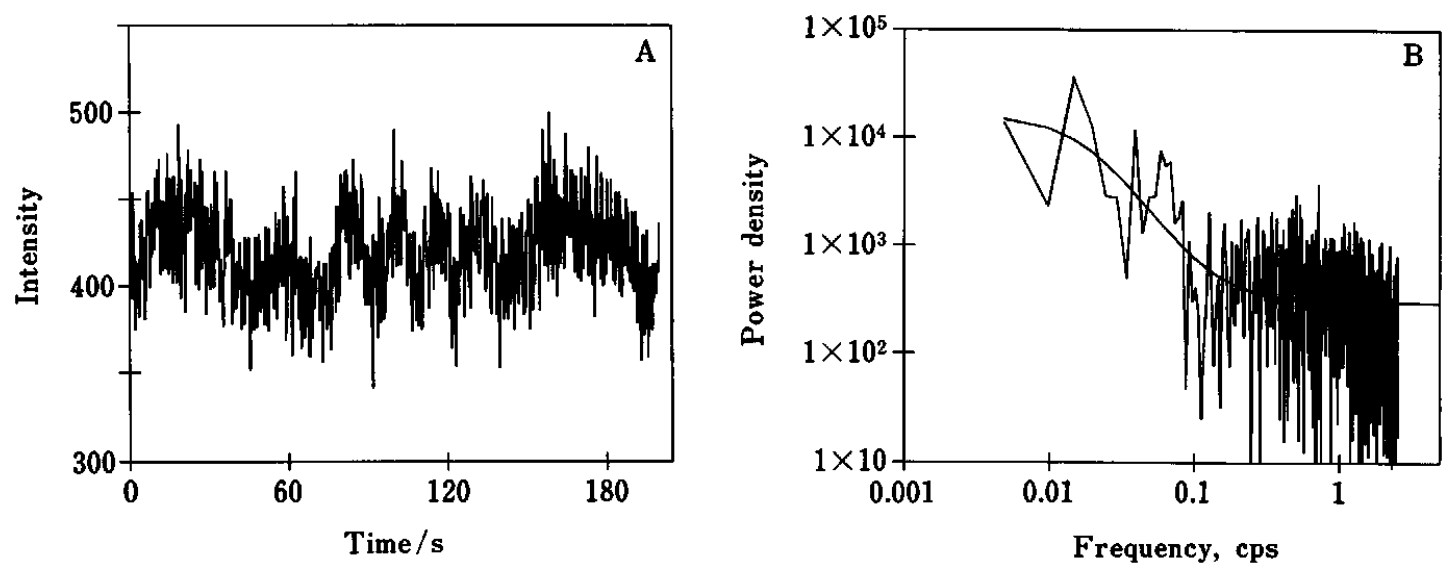

Fig. 4 Blank line and its power spectral density in fluorometry. The number of data points of $A$ is 1024 and that of B 512. The smooth line of B is drawn with the noise parameters, $\widetilde{w}=17.3, \widetilde{m}=5.57$ and $\rho=0.955$. 


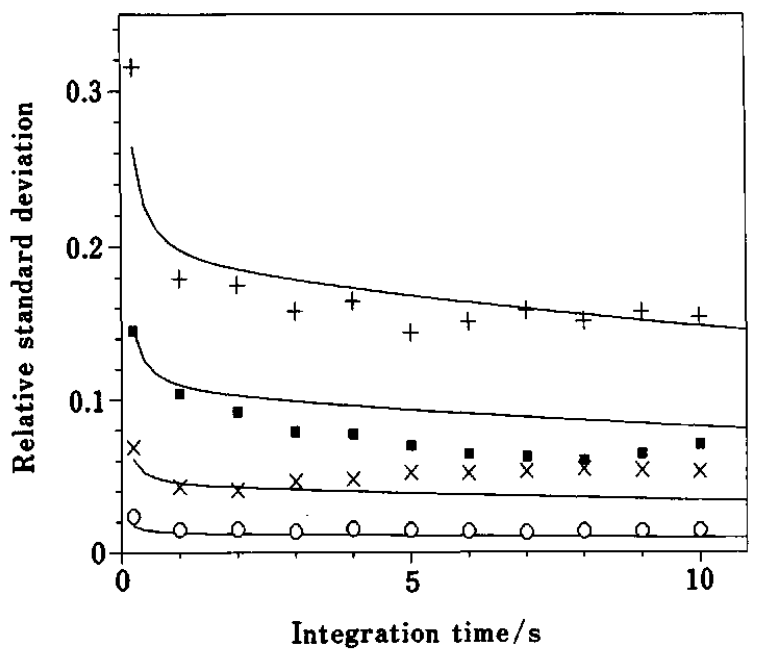

Fig. 5 Observed precision as a function of integration domain in fluorometry. The abscissa denotes the time domain over which the raw data are integrated $\left(=\left(k_{\mathrm{f}}-k_{\mathrm{c}}\right) \times 0.2 \mathrm{~s}\right)$. The solid lines were obtained by Eq. (1). Symbols denote the results from the experiments. $k_{\mathrm{c}}=15$ data points $(=3 \mathrm{~s})$. $0: 2 \mu \mathrm{g} / 1(h=1358) ; \times: 0.5 \mu \mathrm{g} / 1(h=392) ; \square: 0.2 \mu \mathrm{g} / 1(h=163)$; $+: 0.1 \mu \mathrm{g} / \mathrm{l}(h=90.3)$. The noise parameters in the legend of Fig. 4 are used for the prediction. $n=10$ except for the RSDs for the sample of $0.5 \mu \mathrm{g} / \mathrm{l}(n=9)$ where data of anomaly were eliminated from the statistical calculation.

clear presentation, a part of the 4096 point data (1024 point data) are used in Fig. 4A and 512 point data in Fig. 4B. The quality of fit in the power spectrum is satisfactory in spite of the different data.

The power spectrum of the blank line in Fig. 4B shows a typical example of the mixed random process of white noise and the Markov process. The power density of the white noise is horizontal as mentioned above and its contribution can be recognized at high frequencies around $1 \mathrm{cps}$. The negative slope of the power density spotted at low frequencies originates from the Lorentzian shape of the Markov process power spectrum. The mixed process in turn represents the appearance of the $1 /$ $f$ noise. In addition, although the random behavior of the blank drift varies from observation to observation in time space, the power spectra in frequency space always resemble each other, unless the laboratory circumstances are suddenly changed.

Figure 5 shows the observed (symbols) and theoretical (lines) RSD values of integration measurements at different sample concentrations. Equation (1) is used for the uncertainty prediction. Although there are no arbitrary constants involved in the theory and the noise parameters are all determined uniquely from the separate experiments, the theoretical prediction is excellent. The waiting time is sufficiently long $\left(k_{\mathrm{c}}=15\right.$ data points) and no convex curve like + of Fig. 3 is observed in the real fluoroscence measurement. That is, a longer integration yields measurements of higher precision. The observed RSD values at more than $12 \mathrm{~s}$ deviated from the theoretical lines because of the thermal effect. Here, we stress that the theoretical lines in Fig. 5 were not obtained by the usual least squares curve fitting to the experimental RSD values denoted by the symbols.

The prediction theory might seem not to trace some data in Fig. 5 exactly. According to statistics, however, the scattering of the RSD data is generally much more than the scattering of the average such as the deviation of the averaged integrated signals from the true value. The RSD of the observed RSD of the samples obtained from a population is known to be $0.232(=\sigma)$, if $n=10$. For example, the theoretical RSD at an integration time of $6 \mathrm{~s}$ is $9.1 \%$ (see the second line from top in Fig. 5) and its 95.5\% $( \pm 2 \sigma)$ confidence limit ranges from $4.9 \%$ to $13.3 \%$ $(2 \sigma=4.2 \%)$. The observed value $(=6.5 \%$; see $\square$ of Fig. 5) is involved in the confidence level and can be concluded to coincide with the theoretical value within the experimental error. Note that the negligible difference in the Monte Carlo simulation of Figs. 2 and 3 between theory and practice cannot be attained until the simulation is repeated $\mathbf{5 0 0 0}$ times for each integration domain, but the data of Fig. 5 result from only 10 experiments.

Some readers would suspect that any model would fit into the noisy power spectrum shown in Fig. 4B. Even if a power spectrum of the white noise (horizontal line) is used as a model and if the goodness of fit is satisfactory, the uncertainty prediction would give a wrong result. As mentioned above, this is because the above approach lacks the consideration of the more significant effect of the waves of long cycles. In any case, the theoretical prediction of this paper, if the power spectra of baselines are noisy, is much more exact than the traditional method by repeating the measurements of a sample and calculating the RSD in a statistical way. ${ }^{26}$ This fact will be published soon.

Almeida and Seitz ${ }^{16}$ concluded that the fluctuation of the excitation source (here, xenon arc lamp) limits the precision of fluorescence measurements rather than the bandpass, cell orientation or photomultiplier voltage. The strong correlation between the fluctuations of the source intensity and the fluorescence intensity was observed and the precision of measurements ratioed to the source intensity was better than that of the singlechannel measurements. ${ }^{16}$ This fact corroborates our assumption that the fluctuation of the fluorescence signals has the same stochastic property as the fluctuation of the blank line. The uncertainty prediction can be based on the power density of the blank line, and not on the power density of the real fluorescence change. In Eq. (1), the RSD is equal to the SD of the "fake" area created by the random process, divided by the true signal area.

We expect that the precision of measurements varies from instrument to instrument. However, the theoretical approach of this paper is applicable not only to various kinds of fluorometer but also to many different types of analytical instruments. 


\section{Appendix}

Let us consider a situation where the signal is contaminated by the Markov process described by Eq. (3) alone. The path of the random process from data point 1 to $k_{\mathrm{f}}$ is given as $(\Delta t=1$ and $r(0)=0$ ):

$$
\begin{aligned}
r(1)= & m(1) \\
r(2)= & \rho m(1)+m(2) \\
r(3) & =\rho^{2} m(1)+\rho m(2)+m(3) \\
& \cdots \cdots \\
r\left(k_{\mathrm{c}}\right) \quad & \rho^{k \mathrm{c}-1} m(1)+\rho^{k \mathrm{c}-2} m(2) \\
& +\cdots+\rho m\left(k_{\mathrm{c}}-1\right)+m\left(k_{\mathrm{c}}\right) \\
r\left(k_{\mathrm{c}}+1\right)= & \rho^{k \mathrm{c}} m(1)+\rho^{k \mathrm{c}-1} m(2) \\
& +\cdots+\rho m\left(k_{\mathrm{c}}\right)+m\left(k_{\mathrm{c}}+1\right) \\
& \cdots \cdots \\
r\left(k_{\mathrm{f}}\right) \quad & \rho^{k \mathrm{f}-1} m(1)+\rho^{k \mathrm{f}-2} m(2) \\
& +\cdots+\rho m\left(k_{\mathrm{f}}-1\right)+m\left(k_{\mathrm{f}}\right)
\end{aligned}
$$

The area, $R$, between the random path and abscissa during the integration domain $\left(k_{\mathrm{c}}+1, k_{\mathrm{f}}\right)$ is given as:

$$
\begin{aligned}
R= & \sum_{k=k+1}^{k f} r(k) \\
= & \rho^{k c}\left(1+\rho+\rho^{2}+\cdots+\rho^{k \mathrm{f}-k c-1}\right) m(1) \\
& +\rho^{k c-1}\left(1+\rho+\rho^{2}+\cdots+\rho^{k \mathrm{f}-k \mathrm{c}-1}\right) m(2) \\
& +\cdots \cdots \\
& +\rho\left(1+\rho+\rho^{2}+\cdots+\rho^{k \mathrm{f}-k \mathrm{k}-1}\right) m\left(k_{\mathrm{c}}\right) \\
& +\left(1+\rho+\cdots+\rho^{k \mathrm{f}-k \mathrm{c}-1}\right) m\left(k_{\mathrm{c}}+1\right) \\
& +\left(1+\rho+\cdots+\rho^{k \mathrm{f}-k \mathrm{c}-2}\right) m\left(k_{\mathrm{c}}+2\right) \\
& +\cdots \cdots \\
& +(1+\rho) m\left(k_{\mathrm{f}}-1\right) \\
& +m\left(k_{\mathrm{f}}\right)
\end{aligned}
$$

Since the mean of the white noise, $m(t)$, is zero, the mean of the path area is also zero:

$$
E[R]=0
$$

However, the variance of the path area is positive and finite:

$$
\begin{aligned}
E\left[R^{2}\right]= & {\left[\rho^{k c}\left(1+\rho+\rho^{2}+\cdots+\rho^{k f-k c-1}\right)\right]^{2} \widetilde{m}^{2} } \\
& +\left[\rho^{k c-1}\left(1+\rho+\rho^{2}+\cdots+\rho^{k f-k c-1}\right)\right]^{2} \widetilde{m}^{2} \\
& +\cdots \cdots \\
& +\left[\rho\left(1+\rho+\rho^{2}+\cdots+\rho^{k f-k c-1}\right)\right]^{2} \widetilde{m}^{2} \\
& +\left[\left(1+\rho+\cdots+\rho^{k f-k c-1}\right)\right]^{2} \widetilde{m}^{2} \\
& \left.+\left(1+\rho+\cdots+\rho^{k f-k c-2}\right)\right]^{2} \widetilde{m}^{2} \\
& +\cdots \cdots \\
& +(1+\rho)^{2} \widetilde{m}^{2} \\
& +\widetilde{m}^{2}
\end{aligned}
$$

The sum of the first $k_{\mathrm{c}}$ terms is equal to the third term of Eq. (1) and that of the terms from $k_{\mathrm{c}}+1$ to $k_{\mathrm{f}}$ the second term of Eq. (1). The derivation of the first term of Eq. (1) was given elsewhere. ${ }^{17}$

\section{References}

1. R. A. Day, Jr. and A. L. Underwood, "Quantitative Analysis", Prentice Hall, New Jersey, 1991.

2. Y. Hayashi and R. Matsuda, Adv. Chromatogr., 34, 347 (1994).

3. E. Grushka and I. Zamir, Chem. Anal., 98: 529 (1989).

4. P. W. J. M. Boumans, Anal. Chem., 66, 459A (1994).

5. P. W. J. M. Boumans, J. Anal. At. Spectrom., 8, 767 (1993).

6. P. W. J. M. Boumans, Spectrochim. Acta, 46B, 917 (1991).

7. P. W. J. M. Boumans, Spectrochim. Acta., 46B, 641 (1991).

8. E. D. Prudnikov, J. W. Elgersma and H. C. Smit, J. Anal. At. Spectrom., 9, 619 (1994).

9. E. D. Prudnikov, Fresenius' J. Anal. Chem., 337, 412 (1990).

10. E. D. Prudnikov and Y. S. Shapkina, Analyst [London], 109, 305 (1984).

11. C. T. J. Alkemade, W. Snelleman, G. D. Boutilier, B. D. Pollard, J. D. Winefordner, T. L. Chester and N. Omenetto, Spectrochim. Acta, 33B, 383 (1978).

12. G. D. Boutilier, B. D. Pollard, J. D. Winefordner, T. L. Chester and N. Omenetto, Spectrochim. Acta, 33B, 401 (1978).

13. C. T. J. Alkemade, W. Snelleman, G. D. Boutilier and J. D. Winefordner, Spectrochim. Acta, 35B, 261 (1980).

14. K. L. Ratzlaff, Anal. Chem., 51, 232 (1979).

15. A. Andreoni, R. Cubeddu, S. De Silvestri and P. Laporta, Rev. Sci. Instrum., 52, 849 (1981).

16. M. C. Almeida and W. R. Seitz, Appl. Spectrosc., 39, 84 (1985).

17. Y. Hayashi and R. Matsuda, Anal. Chem., 66, 2874 (1994).

18. H. G. Smit and H. L. Walg, Chromatographia, 8, 311 (1975).

19. T. Musha, "Yuragi no Sekai (The world of fluctuation, in Japanese)”, Kodansha, Tokyo, 1993.

20. L. S. Ettre, Pure Appl. Chem., 65, 819 (1993).

21. G. L. Long and J. D. Winefordner, Anal. Chem., 55, 712A (1983).

22. S. Sato, "Suikeigaku no tehodoki", Nankodo, Tokyo, 1962.

23. Y. Hayashi and R. Matsuda, Chromatographia, 41, 66 (1995).

24. Y. Hayashi and R. Matsuda, Anal. Sci., 10, 725 (1995).

25. M. Hino, "Supekutoru Kaiseki", Asakura Shoten, Tokyo, 1982.

26. Private communication, Dr. R. B. Poe (National Institute of Health Sciences), July 10, 1995.

(Received July 14, 1995)

(Accepted October 13, 1995) 